\title{
The relationship between international trade and non-nutritional health outcomes: a systematic
}

review of quantitative studies

Darren K. Burns ${ }^{\mathrm{a}}$, Andrew P. Jones ${ }^{\mathrm{b}}$, Marc Suhrcke ${ }^{\mathrm{a}, \mathrm{c}}$

a. Health Economics Group, Norwich Medical School, University of East Anglia, Norwich, NR47TJ, UK

b. Norwich Medical School, University of East Anglia, Norwich, NR47TJ, UK

c. Centre for Health Economics, University of York, Heslington, York, YO105DD, UK

\section{Abstract}

Markets throughout the world have been reducing barriers to international trade and investment in recent years. The resulting increases in levels of international trade and investment have subsequently generated research interest into the potential population health impact. We present a systematic review of quantitative studies investigating the relationship between international trade, foreign direct investment and non-nutritional health outcomes. Articles were systematically collected from the SCOPUS, PubMed, EconLit and Web of Science databases. Due to the heterogeneous nature of the evidence considered, the 16 included articles were subdivided into individual level data analyses, selected country analyses and international panel analyses. Articles were then quality assessed using a tool developed as part of the project. Nine of the studies were assessed to be high quality, six as medium quality, and one as low quality. The evidence from the quantitative literature suggests that overall, there appears to be a beneficial association between international trade and population health. There was also evidence of the importance of foreign direct investment, yet a lack of research considering the direction of causality. Taken together, quantitative research into the relationship between trade and non-nutritional health indicates trade to be beneficial, yet this body of research is still in its infancy. Future quantitative studies based on this foundation will provide a stronger basis on which to inform relevant national and international institutions about the health consequences of trade policies. 
Keywords

International Trade; Foreign Direct Investment; Population Health; Systematic Review; Study Quality Assessment; Heterogeneous evidence 


\section{Introduction}

Recently, the implications of international trade, or trade in capital, goods and services between nations, to health and health systems have received considerable attention (Blouin et al., 2009; Fidler et al., 2009; Hawkes, 2005; Hawkes \& Thow, 2008; Lee et al., 2009; Owen \& Wu, 2007; Smith et al., 2009c; Walls, 2013). Much of this has been in the form of reports and books from international bodies (Blouin et al., 2006; WHO, 2014), qualitative, and case-study evidence covering a broad range of possible associations between trade and health (Blouin et al., 2009; Hawkes et al., 2009; Smith et al.; Smith et al.; Smith et al.). On the other hand, quantitative research has been chiefly focused on two areas. First has been the relationship between overall patterns of flows in international trade or Foreign Direct Investment (FDI), defined as the inflow of investment to acquire a lasting management interest in an enterprise operating in a different economy from the investor (World Bank, 2014), and levels of international trade in health-related services (Smith, 2004; Smith et al., 2009a; Smith et al., 2009b; Smith et al., 2009c). Secondly, some research has been focused toward associations between flows of FDI, nutrition and other health behaviours including smoking (De Vogli et al., 2014; Gilmore \& McKee, 2005; Hawkes, 2005; Walls, 2013).

The empirical relationship between trade, FDI and more general health outcomes (e.g. mortality) has received comparatively little attention (Moore et al., 2006; Owen \& Wu, 2007). Ostensibly, this work provides a mixed picture as to whether and how trade or FDI affect non-nutritional health outcomes (referred to henceforth just as health). For instance, some evidence has indicated that greater volumes of international trade are associated with increased life expectancy, particularly in low and middle income countries (LMIC's) (Owen \& Wu, 2007). By contrast, other work has suggested that there is no evidence of an association when limiting countries included to those outside the Organization for Economic Co-operation and Development (OECD) (Gerring \& Thacker, 2008) Not only does there appear to be mixed evidence on the relationship itself, the topic is covered by a wide range of different research disciplines. Resultantly, there are notable differences in study setting and 
design throughout available empirical research. We believe there is a need to provide a systematic, comprehensive picture of the existing evidence with a focus on quantitative evidence, the limitations to our understanding and future research opportunities.

To our knowledge, two other relevant systematic reviews currently exist. One focuses on FDI with respect to trade in health services (Smith, 2004), while the other, still in the protocol stage, reviews international food-related trade policy with respect to nutritional health outcomes (Walls, 2013). In this paper we present a systematic review of quantitative evidence on the relationship between international trade or FDI and health outcomes other than those related to nutrition. Due to the nature of the evidence, we develop a strategy to assess the meaning and quality of the heterogeneous evidence by building on previous systematic review methodologies to aid comparability and transparency. The objectives of this article are 1) to identify quantitative research on the relationship between international trade or FDI and non-nutritional population health outcomes, 2) to devise and implement a method to systematically review the literature in the context of varying inputs, methodologies and outcome measures, and 3) to highlight the most important issues raised by the literature and derive recommendations for future research. 


\section{Methods}

We conducted a systematic literature search for relevant papers published up until the end of 2014, using a set of relevant databases (SCOPUS, PubMed, EconLit and Web of Science). Grey literature was searched using Google Scholar. The search strategy was designed to capture any article using trade related, disease or health outcome terms, as well as terms pertaining to relationships between variables or statistical methodologies. Full details of the search strategy are given in Appendix A, and are briefly summarised here:

To be included, papers must have been published up until the end of 2014. Included papers must have included analysis of quantity, change or type of trade in relation to human public health outcome measures including disease prevalence, reported Health Related Quality of Life (HRQoL), mortality, recorded health related events including hospitalisations, or life expectancy. Articles identified as theoretical frameworks, descriptive study designs or those that did not utilise statistical data analysis to test hypotheses made were excluded. Papers discussing trade in relation to obesity, nutritional intake or health behaviours were also excluded as this area was covered by the previous review. Articles not published in the English language were excluded.

As included studies were allowed to vary by data source and analysis method, we expected heterogeneous evidence. The data extracted from each study was as follows: primary author and publication year, study aim, study design, statistical analysis method(s), exposure(s) considered, outcome measures, confounders controlled for and summary results. Extracted summary results included estimated effect sizes with uncertainty measures where applicable. However, it is common in some fields to present multiple model results using different methodologies and variables. In these cases, use of a single numerical result with an uncertainty measure to describe the result of the paper could misrepresent the research. Where this was felt to be an issue, descriptive summary results were provided. 
We devised and implemented a method to systematically review the literature in the context of varying inputs, methodologies and outcome measures. This was achieved using strategies derived from risk of bias assessment of clinical trials and quasi-experimental observational studies. At the time of conducting the review, no single guideline or checklist on risk of bias assessment or quality assessment was found to adequately cover the expected range of study designs. Our approach was therefore to develop a tool which could assess all study designs encountered. We developed this assessment framework based on a combination of existing guidelines and previous review protocols. These were the Cochrane Handbook for Systematic Reviews (Higgins, 2011), guidance from the Campbell Collaboration (Campbell Collaboration, 2009), guidelines from the International Development Consulting group (IDCG) within the Campbell Collaboration (Campbell Collaboration, 2013), and an adapted form of the Campbell Collaboration International Development Coordinating Group (IDCG) guidelines used for a particular review (Baird, 2013; Woolcock et al., 2013). These sources were utilised as they collectively covered the expected study designs. Further, their contribution to the development of the quality assessment tool helped to highlight important methodological, data and results presentation issues in studies regardless of data type, methodology or hypothesis.

The quality assessment tool is available in Appendix B. The five domains of assessment were data quality and collection (D1), data treatment and analysis method (D2), presentation of results (D3), post estimation testing and analysis reporting (D4) and other issues (D5). Domains were selected to cover the full range of data types, approaches, analysis methods, post estimation tests and issues with reporting in the included literature. The fifth domain was required to capture any potential matters that were unique to one research project or not captured by the other domains.

Quality assessment involved assigning a high, medium or low quality rating to each paper, with the lowest score carrying over to the overall score of the article. This approach was based on an adaption of Figure 8.7a in the Cochrane Handbook (Higgins, 2011). Similarly to the IDCG guidelines and 
derivatives, the assessment tool was formatted as a set of questions about the article. A judgement was made in each domain based on answers and any other relevant issues detected. Once each study was examined, an adapted form of Figure 8.7a from the Cochrane Handbook (Higgins, 2011) (Table 1) was used as a decision aid to make judgement of overall quality. As a final check of consistency, $10 \%$ of abstracts were re-screened by one other author and discrepancies in this were discussed before making adjustments.

An initial scoping review of studies suggested that heterogeneity in study designs and outcomes reported rendered it inappropriate to attempt a formal meta-synthesis. Therefore, a narrative analysis of the common themes, results and inferences made was conducted.

Table 1. Cochrane Handbook decision aid, adjusted to our specific purposes

\begin{tabular}{|l|l|l|l|}
\hline Quality & Interpretation & Within a study & Across studies \\
\hline High quality. & $\begin{array}{l}\text { No detected issues, or } \\
\text { issues unlikely to } \\
\text { seriously alter the } \\
\text { results. }\end{array}$ & $\begin{array}{l}\text { High quality for all key } \\
\text { domains. }\end{array}$ & $\begin{array}{l}\text { Most information is from } \\
\text { studies with high quality. }\end{array}$ \\
\hline Medium quality. & $\begin{array}{l}\text { Issues that raise some } \\
\text { doubt about the } \\
\text { internal validity of the } \\
\text { study. }\end{array}$ & $\begin{array}{l}\text { Medium quality for one } \\
\text { or more key domains. }\end{array}$ & $\begin{array}{l}\text { Most information is from } \\
\text { studies at High or } \\
\text { medium quality. }\end{array}$ \\
\hline Low quality. & $\begin{array}{l}\text { Issues detected } \\
\text { seriously weaken } \\
\text { confidence in the } \\
\text { internal validity of } \\
\text { results. }\end{array}$ & $\begin{array}{l}\text { Low quality for one or } \\
\text { more key domains. }\end{array}$ & $\begin{array}{l}\text { The proportion of } \\
\text { information from studies } \\
\text { at low quality is sufficient } \\
\text { to affect the } \\
\text { interpretation of results. }\end{array}$ \\
\hline
\end{tabular}




\section{Results}

\section{Study selection and grouping of evidence}

Application of the search strategy yielded a total of 15,037 results after removal of duplicate titles.

The titles of all these papers were reviewed [D. K. Burns] and 405 papers were put forward for abstract or full paper screening. Once the inclusion and exclusion criteria were applied, 16 papers were accepted for review. Figure 1 summarises the paper screening process. The 16 included studies varied significantly in study design, study setting, and the variables being analysed (Table 1).

However, the range of publication years was narrow. All articles were published between 2006 and 2013.

The differences between articles led to the separation of the evidence into three internally more homogenous categories. Firstly, three studies investigated the relationship between international trade and health using individual level data and these formed Group 1 (Cross et al., 2009a; Cross et al., 2009b; Kawachi, 2008). Three studies were included in Group 2. These studies included data at country level, but included only countries selected in order to highlight a specific issue or test the effect of a discrete event (Bozorgmehr \& Sebastian, 2014; Gustafsson \& Ramstedt, 2011; Oster, 2012). Finally, the remaining 10 studies used a global panel or cross sectional data, and formed Group 3 (Alsan et al., 2006; Azemar \& Desbordes, 2009; Bergh \& Nilsson, 2010; Jorgenson, 2009b; Levine \& Rothman, 2006; Martens et al., 2010; Moore et al., 2006; Owen \& Wu, 2007). Four of these included only LMIC's, and the other six included globally representative sets of countries from all income categories. 


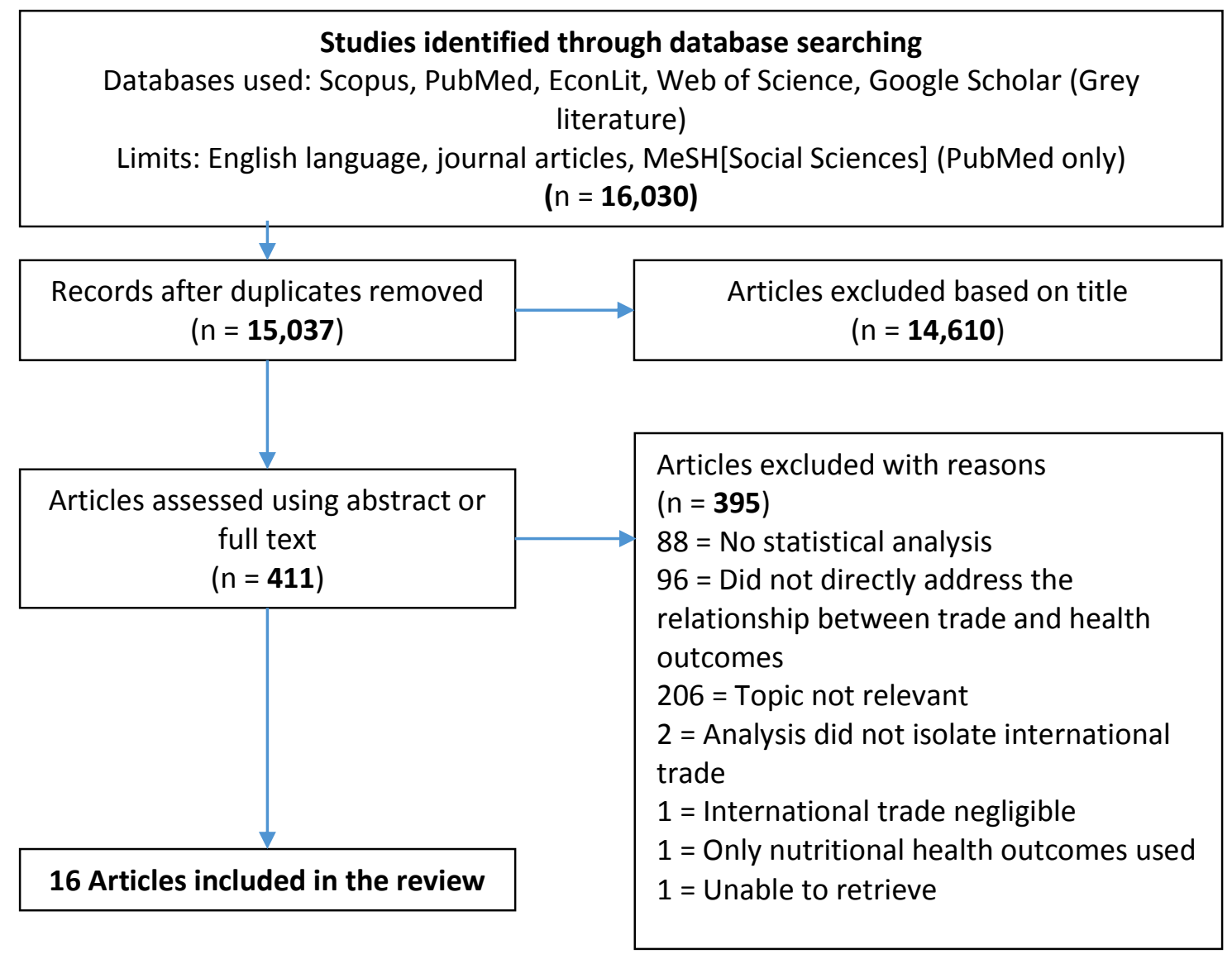

Figure 1. PRISMA diagram of screening process

\section{Review of Evidence}

Below we provide a brief narrative synthesis of the body of literature reviewed, combined with a concise commentary on quality assessment of the articles. More detailed information on the evidence is available in the supplementary data extraction table. A more detailed assessment of study quality can also be found in appendix $C$.

Individual level data analyses (Group 1)

Two studies by Cross et al. used primary data to compare the reported HRQoL of wealthy European countries' horticultural workers with that of workers producing goods for export in African countries 
(Studies 1 and 2 in Table 2) (Cross et al., 2009a; Cross et al., 2009b). The primary analysis method in both was a combination of t-tests and ANOVA. Both studies also used Ordinary Least Squares regression (OLS) to determine whether confounding was an issue. The first study by Cross et al. found that workers producing entirely for export horticulture in Kenya had significantly higher reported HRQoL than those in European countries, suggesting a beneficial statistical association between international trade and health (Cross et al., 2009b). The second study by Cross et al. supported this evidence when comparing Ugandan agricultural workers with those of the UK (Cross et al., 2009a). Both works also suggested that the relationship between export horticultural work and HRQoL was clearer in African samples. However, quality assessment identified the two studies as providing low and medium quality evidence respectively, based on quality of data, method and inference (D1, D2 and D4). Recruitment was not standardised in the different groups considered, raising the suspicion that the evidence was heterogeneous by location.-Further, the HRQoL surveys used (chiefly the SF36) were not designed based on the health perceptions of African populations. As these surveys have not been used to establish population norms in African countries, and tests of consistency between African and European populations have not been carried out to our knowledge, it is unknown whether this had an impact on the internal validity of the study. Population norms for the USA were used in both studies in an attempt to find a common comparator. However, it is not unreasonable to suggest that US health perceptions more closely resemble those of Europeans, raising the possibility of an unreliable point of comparison. The study designs of both studies were not considered to adequately adjust for the heterogeneity discussed above (D2). Further, Ordinary Least Squares (OLS) was used to establish the associations between HrQoL and a set of confounding variables, yet the final study design did not adjust for their confounding effects. Cross et al. therefore presented relatively weak evidence that international trade, or labour in periphery to international trade was related to improved worker health in African countries.

Kawachi used individual level data from the Korean Labour and Income Panel's $4^{\text {th }}$ wave in 2001 to construct a propensity score matching estimator for poor self-reported health by workers under 
either full time or flexible work contracts (Study 3) (Kawachi, 2008). Kawachi argued that globalisation and trade openness were associated with increases in the prevalence of flexible contracts. Results indicated that those with flexible contracts were approximately 1.5 times more likely to report poor health than others with matched characteristics. The quality of the evidence provided was assessed as medium based on the relatively small sample and very brief data description (D1), post-estimation testing and inference (D4). Overall, the study provided fairly robust evidence of an indirect harmful association between trade openness and the health of workers in South Korea in 2001.

Selected country panel analyses (group 2)

The three selected country analyses differed from in study design and setting. Bozorgmehr and Sebastian used data from 22 high Tuberculosis (TB) prevalence countries between 1990 and 2010 to examine evidence for a relationship between economic globalisation and TB incidence (Bozorgmehr \& Sebastian, 2014). OLS, fixed effects and random effects models were used for the analysis in order to both provide comparable results, and adjust for unobserved heterogeneity. In most models, no association was found between measures of economic globalisation (the recently developed Konjunktur-Forschungsstelle Index of Globalisation (KOF), economic domain (KOF1) (Swiss Institute of Technology, 2014), and the Economic Freedom of the World index (EFI) Freedom to Trade Internationally (EFI4) (Berggren \& Jordahl, 2005; Gwartney et al., 2008; Miles et al., 2006)) and TB incidence. However, one model adjusting for time trends provided evidence that an increase of 1 point in the EFI4 was associated with a 10.4\% drop in TB incidence. Increases in KOF1 were associated with a similar magnitude of decline in TB incidence. The authors concluded that no association was present between globalisation and TB incidence, and that the positive results they did find were not sufficiently reliable to infer an association. 
Quality assessment indicated Bozorgmehr and Sebastian to provide high quality evidence. The author was clear that study generalizability was limited, and no data issues were detected (D1). The range of analysis methods used was reasonable (D2), and there was no indication that any results or inference was omitted (D3, D4).

Gustafsson and Ramstedt investigated the removal of personal alcohol import quotas in Sweden Finland and Denmark, and large alcohol tax decreases in both Denmark and Finland (Gustafsson \& Ramstedt, 2011). The outcomes considered were hospitalisations due to acute alcohol poisoning and drink driving, and arrests regarding violent assaults. Generally, no association was found between the policy changes and any of the outcomes. However, a positive association between the Swedish quota increase and hospitalisations due to acute alcohol poisoning was found in those aged 50-69. Quality assessment indicated this evidence to be of a high quality. The data used was adjusted for seasonal change and linearly interpolated across discrete events like riots (D1). The analysis method was a lagged, integrated model using year-on-year change in the variables in question, which raised no suspicions regarding internal validity issues (D2), and the results were thoroughly presented and critically discussed (D3, D4).

Oster used data from 36 sub-Saharan African countries from 1985 to 2007 to investigate whether export of goods was associated with HIV incidence (Oster, 2012). Oster hypothesised that HIV had spread via truck driving, and thus land-based export or import was one mechanism through which international trade could be associated with HIV spreading between African countries. When controlling for country and time fixed effects, a positive association was found between the amount of exports and estimated HIV incidence rates, with a doubling of exports being associated with between a $10 \%$ and $100 \%$ increase in incidence. Quality assessment indicated this evidence to be of high quality. Flaws in the data were carefully considered and discussed (D1), the analysis methodology was seen as appropriate (D2), and Oster adjusted for the effect of a range of confounding variables (D1 D2 D4). 
International panel analyses (group 3)

Five studies investigated relationships between amount of, or dependency on, international trade or trade openness with respect to health (Bergh \& Nilsson, 2010; Gerring \& Thacker, 2008; Levine \& Rothman, 2006; Martens et al., 2010; Owen \& Wu, 2007). All provided evidence to suggest a beneficial association between international trade, or trade dependency and health.

Quality assessment indicated three of these as medium quality (Bergh \& Nilsson, 2010; Martens et al., 2010), and another three as high quality evidence (Gerring \& Thacker, 2008; Levine \& Rothman, 2006; Owen \& Wu, 2007). The studies by Levine and Rothman, Owen and Wu, and Gerring and Thacker, all used similar international panel data sources, which were felt to have a high level of geographical and temporal coverage. Owen and $\mathrm{Wu}$, and Gerring and Thacker, both used fixed effects models to adjust for unobserved heterogeneity between countries, whilst Levine and Rothman went a step further, also adjusting for suspected endogeneity, and testing the robustness of the results when using alternative globalization indices. In all three cases, the approach was considered appropriate, leading to high quality scores for both data (D1) and approach (D2, D4). Finally, none of these studies claimed to establish causal associations, and it is stated that they simply present robust evidence that non-nutritional population health tends to be better in countries more engaged in international trade.

Bergh and Nilsson dropped a significant proportion of their panel to conduct the primary analysis, and this was felt to affect the quality of the data due to a lack of international representativeness (D1). However, in all other aspects the evidence was felt to be of a high quality. Martens et al. was judged to have issues affecting internal validity relating to the choice of analysis method, presentation of results and robustness checking (D2, D3 and D4). The analysis method was crosssectional OLS, and there was no reference to unobserved heterogeneity in the data from different countries, raising suspicions of biased estimation. Further, the choice to present only bivariate 
regressions for the individual effects of index domains was an issue with results presentation (D3), but only with respect to this review which is chiefly concerned with the 'economic globalization' domain. Finally, Martens et al. does not test whether using an alternative globalization index led to similar results. We consider comparison between globalisation indices to be an important robustness check, and other work has highlighted the importance of this (D4) (Zinkina et al., 2013).

Moore et al. investigated the relationship between 'world system role' and infant mortality, finding some evidence of a harmful association (Moore et al., 2006). These roles were allocated to countries satisfying criteria relating to international trade levels, economic globalization and industrial composition of production. The analysis used dummy variables for allocated roles, controlling for geographical and other confounding variables to provide evidence for an association between the type of international trade a country is engaged in and population health. This indicated that 'periphery', or highly specialised and foreign capital dependant countries also had higher levels of infant mortality when controlling for confounders. This article focused on stating the importance of context when investigating international trade and population health, rather than demonstrating particular causal mechanisms through which trade may affect health. Quality assessment indicated this to be high quality evidence. One issue was the choice of an OLS estimation method, yet this was felt to be appropriate considering the necessity to use time invariant dummy variables to capture the 'world system role' (D2). Unobserved heterogeneity was possibly an issue in the results, but internal validity was unlikely to be seriously compromised by this due to the large samples and geographical control variables used.

Four studies either analysed links between FDI and health through a proxy of water pollution, or analysed the effect of health in one year on FDI inflows in subsequent years (Alsan et al., 2006; Azemar \& Desbordes, 2009; Jorgenson, 2009b). The articles by Jorgenson are two similar analyses utilising fixed-effects regression to reveal a significant positive association between FDI in secondary sector industries and water pollution in Low and Middle Income Countries (LMIC's) (Jorgenson, 
2009a, b). Water pollution was separately found to be significantly positively associated with child and infant mortality levels. This evidence suggested one pathway which may explain a harmful relationship between FDI and mortality. It also highlighted the potential importance of industrial composition. However, quality assessment indicated that both studies provided medium quality evidence based on quality of data (D1). The sample size of models including different control variables varied significantly, bringing into question representativeness to all LMIC's. Nevertheless, the fixed effects estimation methods were felt to be appropriate, and there is extensive discussion of confounding. Overall, when considering the paucity of data on industrial composition of FDI to LMIC's, Jorgenson's findings were still considered to be important.

The final two studies, by Alsan et al. and Desbordes and Azemar, provided evidence suggesting that FDI inflows were positively associated with mortality and disease prevalence in LMIC's (Alsan et al., 2006; Azemar \& Desbordes, 2009). Both used international panels representative of LMIC's, and included a range of important confounding variables, leading to a score of high quality in the data domain (D1). The two studies primarily utilised fixed effects estimation also including time dummy variables to adjust for both time specific effects and country specific effects. This was a reasonable approach (D2). Both studies provided a list of countries included, discussed extensively the confounding variables included, and suitably cautioned the interpretation of their results (D3 and D4). 
Table 2: Summary table for study quality assessment

\begin{tabular}{|c|c|c|c|c|c|c|c|c|}
\hline Article & Study & Group & $\begin{array}{l}\text { Data } \\
\text { (D1) }\end{array}$ & $\begin{array}{l}\text { Method } \\
\text { (D2) }\end{array}$ & $\begin{array}{l}\text { Results } \\
\text { (D3) }\end{array}$ & $\begin{array}{l}\text { Analysis } \\
\text { (D4) }\end{array}$ & $\begin{array}{l}\text { Other } \\
\text { (D5) }\end{array}$ & Overall \\
\hline Cross 2009 & 1 & 1 & L & M & $\mathrm{H}$ & $\mathrm{H}$ & N/A & L \\
\hline Cross 2009(1) & 2 & 1 & M & $M$ & M & M & N/A & M \\
\hline Kawachi 2008 & 3 & 1 & $M$ & $\mathrm{H}$ & $\mathrm{H}$ & $M$ & N/A & $M$ \\
\hline Bozorgmehr 2013 & 4 & 2 & $\mathrm{H}$ & $\mathrm{H}$ & $\mathrm{H}$ & $\mathrm{H}$ & N/A & $\mathrm{H}$ \\
\hline Gustafsson 2010 & 5 & 2 & $\mathrm{H}$ & $\mathrm{H}$ & $\mathrm{H}$ & $\mathrm{H}$ & $\mathrm{H}$ & $\mathrm{H}$ \\
\hline Oster 2012 & 6 & 2 & $\mathrm{H}$ & $\mathrm{H}$ & $\mathrm{H}$ & $\mathrm{H}$ & N/A & $\mathrm{H}$ \\
\hline Alsan 2006 & 7 & 3 & $\mathrm{H}$ & $\mathrm{H}$ & H & $\mathrm{H}$ & $\mathrm{N} / \mathrm{A}$ & $\mathrm{H}$ \\
\hline Bergh 2010 & 8 & 3 & M & $\mathrm{H}$ & $\mathrm{H}$ & $\mathrm{H}$ & N/A & M \\
\hline Desbordes 2008 & 9 & 3 & $\mathrm{H}$ & $\mathrm{H}$ & $\mathrm{H}$ & $\mathrm{H}$ & $\mathrm{H}$ & $\mathrm{H}$ \\
\hline Jorgenson 2009 & 10 & 3 & M & $\mathrm{H}$ & $\mathrm{H}$ & $\mathrm{H}$ & N/A & M \\
\hline Jorgenson 2009(1) & 11 & 3 & M & $\mathrm{H}$ & $\mathrm{H}$ & $\mathrm{H}$ & N/A & M \\
\hline Levine 2006 & 12 & 3 & $\mathrm{H}$ & $\mathrm{H}$ & $\mathrm{H}$ & $\mathrm{H}$ & N/A & $\mathrm{H}$ \\
\hline Martens 2010 & 13 & 3 & $\mathrm{H}$ & M & $\mathrm{M}^{*}$ & $\mathrm{H}$ & N/A & M \\
\hline Moore 2006 & 14 & 3 & $\mathrm{H}$ & $\mathrm{H}$ & $\mathrm{H}$ & $\mathrm{H}$ & $\mathrm{H}$ & $\mathrm{H}$ \\
\hline Owen 2007 & 15 & 3 & $\mathrm{H}$ & $\mathrm{H}$ & $\mathrm{H}$ & $\mathrm{H}$ & N/A & $\mathrm{H}$ \\
\hline Gerring 2008 & 16 & 3 & $\mathrm{H}$ & $\mathrm{H}$ & $\mathrm{H}$ & $\mathrm{H}$ & $\mathrm{H}$ & $\mathrm{H}$ \\
\hline
\end{tabular}

*Quality in this domain was only with respect to the topic of this review

Judgements: $L=$ Low study quality. $M=$ Medium study quality. $\mathrm{H}=$ High study quality

Groups: 1 = Individual level data analyses. 2 = Selected country analyses. 3 = International panel analyses.

\section{Discussion}

\section{Summary of results}

The majority of articles included in this systematic review indicate that countries with higher levels of international trade also appear to have better population health. However, the direction and magnitude of this association did vary with the study design, the geographic scale and whether the research focused on international trade in general, or international investments.

In seven out of ten global panel analyses, evidence was provided to suggest that countries with higher levels of international trade, FDI inflows or higher scores on globalisation indices had better population health when adjusting for confounding factors. This evidence was considered to be 
generally reliable as most of these studies presented high quality evidence. In Group 2 studies, the associations between trade and health were less clear, yet the evidence remained of a generally high quality. Two studies found no association between trade and health overall, and the third study provided evidence of one channel through which international trade could be linked with the spread of communicable disease. Finally, Group 1 studies provided evidence of a generally lower quality and did not provide a clear message. Cross et al. provided very weak evidence of labour working for internationally trading firms also having higher HRQoL, while Kawachi provided medium quality evidence to suggest that people working in jobs typically associated with globalised economies have inferior health.

This evidence remained somewhat mixed when only considering high quality studies, though the majority of those studies still found beneficial associations. Moore et al. reported the potential for a harmful association between international trade and health and Oster indicated that the spread of disease between countries in Africa could be related to the export of goods.

Studies indicating harmful associations between trade, or alternatively FDI, and health generally used more specific study settings to those finding only beneficial effects. Five of six studies with the most generalizable study setting, global panels of as many countries as data would allow, indicated a beneficial relationship between levels of trade or FDI and health (Bergh \& Nilsson, 2010; Gerring \& Thacker, 2008; Levine \& Rothman, 2006; Martens et al., 2010; Owen \& Wu, 2007). On the other hand, evidence from articles including only LMIC's more often reported mixed results, but research indicating harms was limited to the impact of FDI (Jorgenson, 2009a, b). Studies selecting countries based on disease prevalence or discrete policy change criteria tended to either find no association, or harmful health associations only in specific demographic groups (Bozorgmehr \& Sebastian, 2014; Gustafsson \& Ramstedt, 2011). Finally, individual level data analyses, which also tended to have the most specific study settings, showed mixed results, and were also generally of a lower quality (Cross et al., 2009a; Cross et al., 2009b; Kawachi, 2008). 
When consolidating the evidence, mixed findings may have been partially due to variation in study setting, study design and input/output variables considered. Despite the heterogeneity in the findings, four messages stood out as potentially usefully informing future work. Firstly, the importance of foreign direct investment as a potential determinant and consequence of health; secondly, the role of sample stratification in affecting the estimated relationship between trade and health in international panel studies; thirdly, the importance of considering mutual association when analysing the trade or FDI and health; and finally, the surprisingly limited use of individual level data. The following sub-section briefly discusses each of these issues.

\section{Review of selected key issues raised by the included literature}

FDI is likely to be a determinant of non-nutritional health, but current evidence is unclear

Two studies found statistically significant associations between health and future FDI inflows (Alsan et al., 2006; Azemar \& Desbordes, 2009), and two others associated FDI in the secondary sector to health outcomes via environmental impact in LMIC's (Jorgenson, 2009a, b). Research using globalisation indices also hinted at a relationship between FDI and health.

High quality evidence suggested that investment flows between countries were affected by health, but evidence suggesting the reverse was constrained by data limitations. Four articles focused their analyses on FDI, rather than international trade in general (Alsan et al., 2006; Azemar \& Desbordes, 2009; Jorgenson, 2009b). Alsan et al. and Desbordes and Azemar provided high quality evidence suggesting that population health affected FDI levels in LMIC's (Alsan et al., 2006; Azemar \& Desbordes, 2009). Jorgenson provided medium quality evidence of an indirect but harmful association between FDI and mortality (Jorgenson, 2009a, b). All four of these studies used panel data representative of LMIC's. 
Bergh and Nilsson, Bozorgmehr and Sebastian, and Martens et al. did not use FDI data directly. Instead they used composite measures of economic globalisation, derived from a combination of FDI, trade and policy data (Bergh \& Nilsson, 2010; Bozorgmehr \& Sebastian, 2014; Martens et al., 2010). Although two of these studies found beneficial associations between the index they used and population health, thereby suggesting the importance of FDI, it was not possible to identify the effect of FDI from the rest of the information used to calculate the index.

The nature or purpose driving investment was also identified as a common theme in the evidence included. Jorgenson analysed FDI specifically into secondary industries, suggesting that FDI in these industries was more likely to be associated with harms to population health (Jorgenson, 2009b). Moore et al. also highlighted the importance of investment orientation (Moore et al., 2006). Countries fulfilling criteria to be allocated to a 'periphery role' were also primarily recipients of secondary sector FDI. These countries saw higher infant mortality figures, and it was suggested that perhaps these two factors were related. Finally, Alsan et al. suggested that the motives driving FDI were a key consideration: the large majority of FDI entering rich industrial countries was to access their markets, rather than for other reasons like manufacturing for export (Alsan et al., 2006). Alsan et al. argued that within LMIC's, which typically see a larger proportion of primary and secondary sector FDI, the association between population health and levels of investment was likely to be stronger.

\section{Sample selection affects the relationship}

The message from this set of evidence was that stratification of countries was important in highlighting important nuances in the relationship between international trade and health. However, two studies indicated that to conduct stratified analysis by income alone may not be sufficient. Instead, research should also look to the nature of the goods being imported or exported, and also the nature (i.e. industry or intention) of international investments. 
Four studies used stratified analysis, and the primary analysis in another was based on separating countries into separate groups based on international trade criteria (Alsan et al., 2006; Bergh \& Nilsson, 2010; Moore et al., 2006; Owen \& Wu, 2007). Owen and Wu provided high quality evidence of a beneficial relationship between trade and life expectancy when only including LMIC's, but no such association among high income countries was found (Owen $\& W u, 2007)$. Despite there being many differences between FDI and international trade, a similar pattern was found by Alsan et al. when looking at the relationship between FDI inflows and life expectancy (Alsan et al., 2006). However, Gerring and Thacker conducted stratified analysis using membership to the OECD, finding no association between international trade and infant mortality in a sample of non-OECD countries (Gerring \& Thacker, 2008).

Bergh and Nilsson repeated the primary analysis many times, changing the inclusion criteria each time (Bergh \& Nilsson, 2010). The country with the highest GDP per capita would be excluded with each iteration. Their results were collated and graphed to give a visual representation of the effect of GDP per capita on the relationship between economic globalisation and health. Generally, the KOF1 index was positively associated with life expectancy. However, when the panel was reduced to mostly lower-middle GDP per capita countries, this relationship appeared to break down. This suggested that there existed a particular income level at which the relationship between trade and health was non-existent, or even harmful.

Finally, Moore et al. revealed a group of eight countries (Kazakhstan, Kenya, Mauritius, Namibia, Senegal, Sri Lanka, Trinidad and Tobago, Zimbabwe) with a potentially harmful association between international trade and mortality, seemingly in concurrence with the continuous stratification approach of Bergh and Nilsson (Bergh \& Nilsson, 2010; Moore et al., 2006). This analysis, along with Gerring and Thacker's model excluding OECD members, highlighted the importance of factors other than national income to the relationship between the trade and non-nutritional health. For example, 
position in the global supply chain, the industries operating within the country, or membership to a global trade network.

The link between trade or FDI and health may be bi-directional.

Twelve articles provided a medium to strong indication of international trade affecting health. Some of these at least considered the possibility of reverse causality, but adjustments for this were typically crude.

Four studies explicitly raised concerns about endogeneity in their models of trade or globalisation affecting non-nutritional health, caused, for instance, by reverse causality (Bergh \& Nilsson, 2010; Gerring \& Thacker, 2008; Levine \& Rothman, 2006; Owen \& Wu, 2007). Bergh and Nilsson, and Gerring and Thacker used lagged explanatory variables to reduce endogeneity bias without exploring the possibility of a two way association, whereas Levine and Rothman and Owen and Wu used study designs that were more robust to endogeneity (Levine \& Rothman, 2006; Owen \& Wu, 2007). Levine and Rothmanused instrumental variables regression to adjust for endogeneity in their primary analysis (Levine \& Rothman, 2006). International trade as a percentage of GDP was instrumented by the estimated trade to GDP ratio based on a 'gravity model' (Frankel \& Romer, 1999).

Owen and Wu tested for reverse causality in an arguably somewhat crude way (Owen \& Wu, 2007), i.e. by simply repeating the primary fixed effects regression analysis of trade openness versus life expectancy, but in reverse. Taken literally, the results indicated that lagged adult life expectancy was not generally significantly associated with trade openness in that direction, but was associated with the import of medical goods and their relative prices. Since these two pieces of evidence were concerned with different health outcomes, they did not entirely contradict one another. However, it remains that only a small number of studies have attempted to actually explore the idea of a mutual 
association between trade and non-nutritional health. Future research should seriously consider the possibility of endogeneity in order to avoid presenting biased and possibly misleading results.

There is evidence for FDI affecting health, health affecting FDI, and some evidence indicating an interrelationship between communicable disease and FDI. The studies by Alsan et al., and Debordes and Azemar, provided high quality evidence to suggest that mortality was significantly negatively related to future inflows of FDI in LMIC's (Alsan et al., 2006; Azemar \& Desbordes, 2009). Conversely, the works by Jorgenson were also focused on FDI in LMIC's, and provided medium quality evidence of a significant harmful association between lagged secondary sector FDI and infant mortality rates (Jorgenson, 2009a, b). Further, Desbordes and Azemar went on to discuss possible endogeneity, yet only between health and income or health and education within the context of the association between HIV prevalence and FDI inflows. Overall, this evidence suggested that FDI and health could be interrelated, but to date there is no empirical evidence to explicitly demonstrate this. Future work on the relationship between FDI and health should try to more specifically examine potential reverse causality.

Individual level data analyses were scarce and had data related limitations, yet still provided important results

Just three of fifteen studies made use of individual level data (Cross et al., 2009a; Cross et al., 2009b; Kawachi, 2008). The focus of these was exclusively on the health of those employed in export horticulture (Cross et al., 2009a; Cross et al., 2009b), or workers under flexible work contracts, typically associated with economies heavily engaged in international trade (Kawachi, 2008). The shortage of individual level data analyses in this field may be related to the difficulty of obtaining appropriate data, particularly in LMIC's. Even when conducting primary data collection as Cross et al. did, data quality issues were present due to the inconsistency of data collection covering many populations around the world (Cross et al., 2009a; Cross et al., 2009b). 
Individual level evidence was - as it should be - highly specific to the populations being analysed. Kawachi associated flexible work contracts with economic globalisation, and this may or may not have been the same for any other country in the world. However, the use of labour survey data also increased the depth with which Kawachi could analyse the South Korean working population (Kawachi, 2008). The same specificity to the populations assessed was also true of Cross et al. (Cross et al., 2009a; Cross et al., 2009b) Of course, this limited level of generalizability was noted by authors of all three studies. Overall, these studies provided original insight into aspects of the international trade and health relationship that could not otherwise be detected.

\section{Review strengths and weaknesses}

To the best of our knowledge, this is the first review with a particular focus on quantitative evidence of international trade affecting non-nutritional outcomes. We have assessed heterogeneous evidence covering various study designs, settings and included variables. Study designs ranging from individual level to country-level global panel analyses were all taken into account during the review and quality assessment processes. We developed a quality assessment tool quite specifically targeted for the literature reviewed here to encompass this range. Use of this tool facilitated the identification of key issues in terms of sensitivity of the results, and recommendations for future research.

A weakness of this study include the lack of quantitative meta-synthesis. This was primarily due to the heterogeneity of the reviewed evidence which precluded the use of the method. Further, the search strategy limited the inclusion of papers to the English language. A (likely) small share of potentially relevant evidence may therefore have been missed. Thirdly, the separation of the evidence into three groups due to lack of comparability, although deemed to be necessary to derive important messages, illustrated the extent of heterogeneity in the evidence being assessed. Finally, there is a wealth of theoretical, descriptive, qualitative and case-study evidence on relationships between international trade and health which were outside the scope of our inclusion criteria 
(Blouin et al., 2009; Blouin et al., 2006; Fidler et al., 2009; Gerring \& Thacker, 2008; Hawkes et al., 2009; Lee et al., 2009; Smith et al., 2009a; Smith et al., 2009c). Other types of evidence are unquestionably of importance to understanding the association between trade and population health. However, we intentionally present systematically retrieved and quality assessed quantitative evidence on the topic. We believe that consideration of evidence from a quantitative perspective is useful in itself, has provided an original insight into the issue, and highlighted several important questions for future research.

\section{Suggestions for future research and policy implications}

First, we see scope for more research on the relationship between FDI and health outcomes. As discussed, there already exists evidence from several studies to suggest that health affects FDI inflows (Alsan et al., 2006; Azemar \& Desbordes, 2009), that FDI in secondary industries affects infant/child mortality through its effect on water pollution (Jorgenson, 2009b), and that the intention and nature of FDI affects how it might impact on health outcomes (Alsan et al., 2006; Jorgenson, 2009b). It may be particularly worthwhile to examine whether the FDI and health relationship differs by health outcome considered (e.g. adult health vs. child health), by the specific industries the FDI is targeting, and by geographical concentration of FDI.

Second, better testing and controls for endogeneity between trade, or alternatively FDI, and nonnutritional health would provide useful insights into the true nature of the relationship between the two. Third, two papers indicated that there may exist a subset of countries with an apparently different relationship between international trade or economic globalisation and health from other countries, even when controlling for major confounders like income (Bergh \& Nilsson, 2010; Moore et al., 2006). We therefore recommend research to establish the reasons for this, including understanding the importance of not only quantity or rules surrounding trade (or FDI), but the composition of it as well. 
Fourth, we were unable to find any quantitative data analysis on non-nutritional health impacts of trade agreements, trade relations or trade politics. Indeed, only Gustafsson and Ramstedt analysed discrete trade policy changes of any kind in relation to non-nutritional health outcomes (Gustafsson \& Ramstedt, 2011). Quantitative research on this topic could reveal that not only the quantity of trade is important to its association with health.

Fifth, we see scope for quantitative data analyses forecasting the potential future impact of trade on non-nutritional health outcomes (and vice-versa). All of the included evidence had a focus on establishing the existence of relationships observed in past data, rather than attempting to estimate the future impacts.

Finally, more individual level analysis on this topic is recommended. The studies by Cross et al. lacked consistency in the data being collected by different institutions, and this led to significant limitations in the resulting evidence. Future research on an individual level should focus on ensuring the quality and consistency of the data being collected. Such a study could isolate particular exposures as particularly damaging or beneficial.

Policy implications

Public health policy makers and advocates need to be aware that international trade may affect a broad range of health outcomes, well beyond the specifically nutrition-related ones that have been the focus of much of the 'trade \& health' literature so far. While overall, trade appears to entail beneficial health effects, there remain examples in various studies of potential adverse health effects, including the spread of infectious disease and increases in pollution resulting in mortality increases amongst children. What follows from the mixed evidence is that relevant institutions (such as the UN or World Bank) need to at least monitor and seek to assess the health impact of trade policies. Should harmful effects be identified (or predicted), policies need to be considered to mitigate or prevent such adverse effects. Finally, healthier countries, particularly healthier countries within sub-Saharan Africa, have been shown to attract more FDI than the less healthy (Alsan et al., 
2006; Azemar \& Desbordes, 2009). Policymakers seeking more FDI, with the likely positive economic effects commonly associated with it, might reasonably see this is as an additional incentive for increasing efforts to improve population health. 


\section{References}

Alsan, M., Bloom, D.E., \& Canning, D. (2006). The effect of population health on foreign direct investment inflows to low-and middle-income countries. World Development, 34, 613-630.

Azemar, C., \& Desbordes, R. (2009). Public Governance, Health and Foreign Direct Investment in SubSaharan Africa. Journal of African Economies, 18, 667-709.

Baird, F.H.G.F., Berk Özler, Michael Woolcock (2013). Relative Effectiveness of Conditional and Unconditional Cash Transfers for Schooling Outcomes in Developing Countries: A Systematic Review PROTOCOL. In C. Collaboration (Ed.).

Berggren, N., \& Jordahl, H. (2005). Does free trade really reduce growth? Further testing using the economic freedom index. Public Choice, 122, 99-114.

Bergh, A., \& Nilsson, T. (2010). Good for Living? On the Relationship between Globalization and Life Expectancy. World Development, 38, 1191-1203.

Blouin, Chopra, M., \& van der Hoeven, R. (2009). Trade and social determinants of health. Lancet, 373, 502-507.

Blouin, Drager, N., \& Smith, R. (2006). International trade in health services and the GATS: current issues and debates: World Bank Publications.

Bozorgmehr, K., \& Sebastian, M. (2014). Trade liberalization and tuberculosis incidence: a longitudinal multi-level analysis in 22 high burden countries between 1990 and 2010. Health Policy Plan, 29, 328-351.

Campbell Collaboration. (2009). Producing a review: Campbell Collaboration.

Campbell Collaboration. (2013). International Development Coordinating Group (IDCG): Protocol and review methods guidance. http://www.campbellcollaboration.org: campbell Collaboration.

Cross, P., Edwards, R.T., Nyeko, P., \& Edwards-Jones, G. (2009a). The potential impact on farmer health of enhanced export horticultural trade between the U.K. and Uganda. International Journal of Environmental Research and Public Health, 6, 1539-1556.

Cross, P., Edwards, R.T., Opondo, M., Nyeko, P., \& Edwards-Jones, G. (2009b). Does farm worker health vary between localised and globalised food supply systems? Environment International, 35, 1004-1014.

De Vogli, Kouvonen, A., Elovainio, M., \& Marmot, M. (2014). Economic globalization, inequality and body mass index: a cross-national analysis of 127 countries. Critical Public Health, 24, 7-21.

Fidler, Drager, N., \& Lee, K. (2009). Managing the pursuit of health and wealth: the key challenges. The Lancet, 373, 325-331.

Frankel, J.A., \& Romer, D. (1999). Does trade cause growth? American economic review, 379-399.

Gerring, J., \& Thacker, S.C. (2008). Do neoliberal economic policies kill or save lives? Business and Politics, 10.

Gilmore, A.B., \& McKee, M. (2005). Exploring the impact of foreign direct investment on tobacco consumption in the former Soviet Union. Tob Control, 14, 13-21.

Gustafsson, N.-K.J., \& Ramstedt, M.R. (2011). Changes in alcohol-related harm in Sweden after increasing alcohol import quotas and a Danish tax decrease-an interrupted time-series analysis for 2000-2007. International journal of epidemiology, 40, 432-440.

Gwartney, J., Lawson, R., \& Norton, S. (2008). Economic freedom of the world 2008 annual report: The Fraser Institute.

Hawkes (2005). The role of foreign direct investment in the nutrition transition. Public Health Nutrition, 8, 357-365.

Hawkes, Blouin, C., Henson, S., Drager, N., \& Dubé, L. (2009). Trade, food, diet and health: perspectives and policy options: John Wiley \& Sons.

Hawkes, \& Thow (2008). Implications of the Central America-Dominican Republic-Free Trade Agreement for the nutrition transition in Central America. Rev Panam Salud Publica, 24, 345360. 
Higgins, J.G., S (editors) (2011). Cochrane Handbook for Systematic Reviews of Interventions Version 5.1.0 The Cochrane Collaboration.

Jorgenson, A.K. (2009a). Foreign Direct Investment and the Environment, the Mitigating Influence of Institutional and Civil Society Factors, and Relationships Between Industrial Pollution and Human Health A Panel Study of Less-Developed Countries. Organization \& Environment, 22, 135-157.

Jorgenson, A.K. (2009b). Political-Economic Integration, Industrial Pollution and Human Health A Panel Study of Less-Developed Countries, 1980-2000. International Sociology, 24, 115-143.

Kawachi, I. (2008). Globalization and workers' health. Ind Health, 46, 421-423.

Lee, K., Sridhar, D., \& Patel, M. (2009). Bridging the divide: global governance of trade and health. The Lancet, 373, 416-422.

Levine, D.I., \& Rothman, D. (2006). Does trade affect child health? Journal of Health Economics, 25, 538-554.

Martens, P., Akin, S.M., Maud, H., \& Mohsin, R. (2010). Is globalization healthy: a statistical indicator analysis of the impacts of globalization on health. Global Health, 6.

Miles, M.A., Holmes, K.R., O'Grady, M.A., Eiras, A.I., Schaefer, B.D., \& Kim, A.B. (2006). 2006 Index of economic freedom: Heritage Foundation.

Moore, S., Teixeira, A.C., \& Shiell, A. (2006). The health of nations in a global context: Trade, global stratification, and infant mortality rates. Social Science and Medicine, 63, 165-178.

Oster, E. (2012). Routes of Infection: Exports and Hiv Incidence in Sub-Saharan Africa. Journal of the European Economic Association, 10, 1025-1058.

Owen, A.L., \& Wu, S. (2007). Is Trade Good for Your Health? Review of International Economics, 15, 660-682.

Smith (2004). Foreign direct investment and trade in health services: A review of the literature. Soc Sci Med, 59, 2313-2323.

Smith, Chanda, R., \& Tangcharoensathien, V. (2009a). Trade in health-related services. The Lancet, 373, 593-601.

Smith, Correa, C., \& Oh, C. (2009b). Trade, TRIPS, and pharmaceuticals. The Lancet, 373, 684-691.

Smith, Lee, K., \& Drager, N. (2009c). Trade and health: an agenda for action. The Lancet, 373, 768 773.

Swiss Institute of Technology. (2014). Konjunktur-Forschungsstelle (KOF) Index. In S.I.o.T. Zurich (Ed.), KOF Index of Globalization.

Walls, H.C., L; Freil, S; Smith, R. (2013). Systematic Review Protocol: A review of the impact of international food-related trade policy on nutrition and health. Licrah database of systematic reviews.

WHO. (2014). Trade and Health. World Health Organization.

Woolcock, M., Özler, B., Baird, S., \& Ferreira, F.H.G. (2013). Relative Effectiveness of Conditional and Unconditional Cash Transfers for Schooling Outcomes in Developing Countries: A Systematic Review.

World Bank. (2014). Definition of Foreign Direct Investment.

Zinkina, J., Korotayev, A., \& Andreev, A.I. (2013). Measuring globalization: existing methods and their implications for teaching Global Studies and forecasting. Campus-Wide Information Systems, 30, 321-339. 